\title{
Aboriginal and Torres Strait Islander Australians and tobacco
}

\author{
V L Briggs, K J Lindorff, R G Ivers
}

\section{Suffering high mortality and morbidity rates from conditions related to tobacco use}

A ustralia is considered to be a leader among developed nations in the area of tobacco control. Well coordinated and sustained tobacco control programmes are likely to have resulted in long term health gains, including a decrease in mortality rates from cardiovascular disease, and subsequent increases in life expectancy.

However, Aboriginal and Torres Strait Islander people-the indigenous people of Australia-continue to suffer high mortality and morbidity rates from conditions related to tobacco use. Indeed tobacco is the main cause of preventable mortality among Aboriginal and Torres Strait Islander Australians. ${ }^{1}$ How is it that a minority group within a wealthy nation can display such marked health status and tobacco use differences from others in that population? What has been done to attempt to redress this disparity? What are the needs and priorities for the future? In this commentary, we describe cultural, historical, and political factors leading to the high prevalence of smoking among Aboriginal and Torres Strait Islander people. We outline and discuss intervention efforts undertaken so far and consider their merits and weaknesses, paying particular attention to resource allocation issues. Finally, we consider priorities for future programme delivery that would be most likely to result in reduced tobacco use among this population.

\section{PREVALENCE OF TOBACCO USE}

As for other indigenous people worldwide, particularly those who have been colonised by other people (for example New Zealand Maori, ${ }^{2}$ native Canadians, and native Americans), the prevalence of tobacco use is higher among Australian Aboriginal and Torres Strait Islander people than for other Australians.

In the National Aboriginal and Torres Strait Islander Survey ${ }^{4}$ conducted in 1994, 54\% of Aboriginal and Torres Strait Islander men and $46 \%$ of Aboriginal and Torres Strait Islander women were smokers, with very few Aboriginal and Torres Strait Islander people being exsmokers. Studies have indicated that in some communities the prevalence of

tobacco use is as high as $83 \%$ among men $^{5}$ and $73 \%$ among women. ${ }^{6}$ By comparison, in 1998 25\% of Australian men and $20 \%$ of Australian women were smokers. ${ }^{\text {? }}$

Although some Aboriginal and Torres Strait Islander people chew tobacco or smoke traditional pipes, as for other Australians ${ }^{8}$ most smoke tailor made or roll your own cigarettes and consume similar quantities to other Australians. ${ }^{4} 7$ Uptake of smoking is often at an earlier age than for other Australians. ${ }^{6}$ Patterns of tobacco use may differ from those for other Australians; many Aboriginal and Torres Strait Islander people share tobacco and smoke sporadically according to availability of money for tobacco.

\section{MORTALITY RELATED TO TOBACCO USE}

The health status of Aboriginal and Torres Strait Islander people is markedly worse than that of other Australians. There is an abundance of information documenting the burden of morbidity and premature mortality experienced by Aboriginal and Torres Strait Islander Australians; in 1992-1994 they had a life expectancy that was 15-20 years less than that of other Australians (75.0 years for males born in 1994 and 80.9 years for females). ${ }^{9}{ }^{10}$ In a model used for calculating life expectancy for 15 year old Australians, regular tobacco use was likely to reduce life expectancy by 6.5 years in males and 5.1 years in females. ${ }^{11}$ Arnold-Reed et al calculated the gain in life expectancy that would be expected in Aboriginal and Torres Strait Islander peoples if additional alcohol and tobacco related deaths were eliminated. ${ }^{12}$ If tobacco related deaths were eliminated, life expectancy for men would increase from 58.5 years to 61 years, and for women would increase from 65.3 years to 67 years. However, modeling of life expectancy is likely to provide conservative estimates of potential increase in life expectancy because other factors such as maternal and childhood malnutrition, and exposure to campfire smoke and dust, may also exacerbate the effects of tobacco use.

Aboriginal and Torres Strait Islander people experience 2-8 times the death rate of others in all age groups, ${ }^{13}$ however, the percentage of deaths attributable to tobacco use is likely to be similar to that in the mainstream populationabout $15 \% .{ }^{14}{ }^{15}$

Aboriginal and Torres Strait Islander people exhibit higher rates of tobacco related diseases such as cardiovascular diseases and pneumonia, cervical cancer, cataracts, and ear disease than other Australians. They are hospitalised at 2-3 times the rate of people in the general Australian population, with respiratory disease-much related to tobacco useand injury being the most common causes of hospitalisation. ${ }^{10}$

\section{REASONS FOR THE HIGH}

\section{PREVALENCE OF TOBACCO USE}

The high level of tobacco use and subsequent harm among Aboriginal and Torres Strait Islander people may be related to a number of factors.

Aboriginal people have long chewed the dry leaves of pituri (Duboisia hopwoodii) and native tobacco such as Nicotania gossei and $N$ excelsior prior to contact with non-Aboriginal people. ${ }^{16}$ Some Aboriginal people continue to use pituri and native tobaccos. Macassan fishermen and trepangers introduced tobacco into northern Australia approximately 400 years ago. ${ }^{17}$ People in this region continue to use Macassan style pipes and tobacco still plays a role in traditional ceremonies. Such traditional use of tobacco may reinforce its use today, although not all Aboriginal and Torres Strait Islander people used tobacco traditionally-for example Aboriginal people in southeastern Australia are unlikely to have had access to tobacco until contact with Europeans.

In some Aboriginal and Torres Strait Islander communities traditional beliefs held by community members may influence their tobacco use or their perceptions of the benefits of quitting. A specific example is that in some traditional Aboriginal and Torres Strait Islander societies people may believe that serious illnesses that are, according to the biomedical model, caused by tobacco are rather caused by sorcery, interpersonal conflict, or the breaking of taboos, which may mean that a cessation message is not seen as believable. However, there is great cultural diversity in Aboriginal and Torres Strait Islander communities and not all will hold such beliefs.

The process of colonisation of Australia meant that many Aboriginal and Torres Strait Islander people came into contact with tobacco through missionaries, miners, fishermen, anthropologists, and cattle station workers. Tobacco was highly valued by Aboriginal and Torres Strait Islander people ${ }^{18}$ and Europeans often used it to manipulate them. ${ }^{19}$ Until 
they obtained Australian citizenship in 1967, Aboriginal and Torres Strait Islander people were confined in missions or worked on cattle stations and were given rations, including tobacco, in exchange for their labour and for attending church-for living a "civilised" lifestyle..$^{20}$ For many elderly Aboriginal and Torres Strait Islander people, their tobacco use commenced when they were given such rations. The process of colonisation is likely to have caused-and continue to cause-higher levels of stress (and subsequent tobacco use). For example "stolen children" - those who were removed from their families by force as children, for example so as to be relocated to missions-exhibit a higher prevalence of tobacco use than others. ${ }^{21}$

The process of colonisation, including the removal of Aboriginal and Torres Strait Islander people from their traditional lands, meant that they now form an underclass within Australia and that there are profound differences in the socioeconomic status of Aboriginal and Torres Strait Islander people compared with that of other Australians. They are more likely to be unemployed than other Australians, and more likely to have a lower median weekly income ${ }^{22}$; as with other Australians, the prevalence of tobacco use is lower among Aboriginal and Torres Strait Islander people who are employed. ${ }^{41}$ Many Aboriginal and Torres Strait Islander people live in substandard housing where overcrowding is a problem, ${ }^{23}$ which means that they are more likely to be exposed to environmental tobacco smoke.

Aboriginal and Torres Strait Islander people are less likely than other Australians to have attained higher levels of education; ${ }^{22}$ as with other Australians, a lower level of education appears to be linked to tobacco use. ${ }^{21}$ The presence of other risk factors, such as poverty, childhood malnutrition, and living in dusty rural or remote environments, may further enhance the health risks of tobacco.

The normalisation of tobacco use within Aboriginal and Torres Strait Islander societies may play a role in ensuring social cohesion among Aboriginal and Torres Strait Islander Australians to a larger extent than it does in mainstream society. Tobacco use reinforces family relationships and friendships. ${ }^{24}$ Non-participation may lead to people feeling isolated and alienated from the group. Aboriginal and Torres Strait Islander people have commented that the presence of other smokers makes it difficult to quit and to maintain cessation. ${ }^{25}$

Lack of knowledge about the health effects of tobacco does not appear to be a major factor contributing to the higher prevalence of tobacco use by Aboriginal and Torres Strait Islander people. Although the 1994 National Drug Strategy survey ${ }^{26}$ found that only $5 \%$ of Aboriginal and Torres Strait Islander people believed that smoking could damage their health, this lack of knowledge about the health effects of tobacco has not been borne out in other surveys. ${ }^{27}$ For example, Aboriginal and Torres Strait Islander people interviewed about the 1997-8 National Tobacco Campaign were well aware that smoking could cause respiratory disease. ${ }^{28}$

Despite apparent adequate knowledge about the potential health effects of tobacco use, Aboriginal and Torres Strait Islander people have generally not identified tobacco as a health issue that should be given priority, for example, compared with alcohol. The effects of tobacco use are "invisible" and chronic; they are not obvious. Similarly, health departments and other agencies do not appear to have prioritised service delivery in this area.

A lack of availability and access to culturally appropriate health services for Aboriginal and Torres Strait Islander people is likely to have contributed to the high prevalence of tobacco use. Services for this population are poorly funded according to need. ${ }^{29}$ There are many barriers for Aboriginal and Torres Strait Islander people when accessing preventive and curative health services, including language barriers and racism. ${ }^{30}$

\section{PROGRAMME DELIVERY}

The delivery of tobacco control programmes in Australia has been characterised by uncoordinated and unsustained funding and delivery. A range of health organisations, including State or Territory health departments, nongovernment agencies, and Aboriginal and Torres Strait Islander community controlled organisations, have attempted to deliver tobacco control programmes to Aboriginal and Torres Strait Islander people.

This lack of sustainable funding, coupled with a fragmented approach, has seen many programmes over the past decade dwindle and ultimately cease; many of these projects had only been funded for a limited period of 12 months or so. State based, rather than national funding for health programme delivery has meant that there has never been a coordinated national approach compared with that which, for example, is used by the Indian Health Service in the United States.

Examples of tobacco control programmes targeted at Aboriginal and Torres Strait Islander Australians include training health professionals to deliver brief advice on cessation. The Queensland Department of Health together with Aboriginal and Torres Strait Islander health workers have developed a brief intervention package that aims to train primary health care workers to give one on one advice and support in the area of tobacco cessation. ${ }^{31}$

Similarly, the Quit campaign in Victoria runs training for Aboriginal and Torres Strait Islander health workers based on and adapted from the Fresh Start cessation programme used in the wider population. ${ }^{32}$ Both of these programmes were based on an adapted version of the Prochaska and Di Clemente stages of behavioural change model. ${ }^{33}$ The Aboriginal and Torres Strait Islander programme seeks to have a capacity building effect by empowering Aboriginal and Torres Strait Islander health workers to develop skills to assist their respective community members to quit smoking.

An audit of existing tobacco control programmes showed that many agencies had developed health promotion material about tobacco targeted at Aboriginal or Torres Strait Islander people. ${ }^{27}$ Despite the many traditional languages in use in Australia, almost none of the health promotion resources were presented in them, although some displayed traditional artwork.

There have been several small unpublished trials of nicotine replacement therapy for Aboriginal and Torres Strait Islander people (V Briggs et al, personal communication, 2002), indicating that nicotine patches may be of use to some Aboriginal and Torres Strait Islander smokers. However, bupropion has not yet been assessed in this population.

Mainstream programmes may have some influence on Aboriginal and Torres Strait Islander people. An evaluation of the National Tobacco Campaign-a national anti tobacco media campaignconducted among Koori people in Victoria showed that campaign messages had increased awareness in this population. ${ }^{28}$ However, the effect of tobacco policy interventions, such as legislation to prohibit smoking in enclosed public places, or the effect of price increases for tobacco, has not been assessed in this population. There has been almost no formal evaluation of tobacco control programmes for this population. ${ }^{27}$ Despite an abundance of research investigating interventions on tobacco in other populations, no such formal research has been conducted in this setting.

\section{FUTURE PROGRAMME DELIVERY}

The National Aboriginal and Torres Strait Islander Tobacco Control Project was undertaken by the National Aboriginal Community Controlled Health Organisation (NACCHO) between November 2000 and June 2002. The project aimed to determine key issues for tobacco control in Aboriginal and Torres Strait Islander communities and make recommendations for future action 
Table 1 Key recommendation and principles for tobacco control in Aboriginal and Torres Strait Islander communities ${ }^{25}$

\section{Key recommendation}

That national coordination for Aboriginal and Torres Strait Islander tobacco control be implemented without delay. Dedicated funding will be required to support this role. Collaboration between and representation from Aboriginal community controlled health, Torres Strait Islander community controlled health, tobacco control, government, and non-government organisations will be necessary. Principles

1. Aboriginal and Torres Strait Islander tobacco control programmes should seek to maximise community control.

2. All individuals and organisations working on programmes in Aboriginal and Torres Strait Islander tobacco control should understand and respect the social context in which Aboriginal people and Torres Strait Islanders live their lives and programmes should reflect this understanding 3. Tobacco control programmes for Aboriginal and Torres Strait Islander communities should be holistic in nature and consider the social determinants of health.

4. Tobacco control programmes for Aboriginal and Torres Strait Islander communities should be as comprehensive as possible within given resources.

based on comprehensive consultation with Aboriginal and Torres Strait Islander community members and health professionals across Australia.

The final report for the project, Tobacco - time for action, ${ }^{25}$ made one overall key recommendation and four overarching principles to guide future work in this area. These are shown in table 1.

Although tobacco control activity in Aboriginal and Torres Strait Islander communities has increased recently, lack of coordination has at times resulted in duplication of effort, meaning already scarce resources are not being used efficiently. Although the examples of health worker training programmes given above were considered to be successful within their respective States, a more nationally coordinated approached would help to ensure that the most effective programmes are implemented and that lessons learned from programme implementation are shared across communities. ${ }^{25}$ The NACCHO report called for urgent, collaborative, national coordination. This is supported by a number of other recent national reports that recognised lack of coordination was linked to lack of progress, action, and priority in Aboriginal and Torres Strait Islander tobacco control. ${ }^{27}{ }^{34}$ Increased coordination may also help to overcome some of the problems associated with geographical isolation and an absence of structured communication networks.

The need to respect the social context of Aboriginal and Torres Strait Islander tobacco use, in particular to acknowledge some of the unique historical and cultural factors, as discussed, leading to this use, is vital if culturally appropriate programmes and support are to be developed and provided to smokers. Participants in the NACCHO project believed that maximising community control of projects would help to ensure that this respect for the social context of tobacco use was acknowledged and that programmes were based on concepts of holistic health. ${ }^{25}$ They stated this meant in reality that Aboriginal and Torres Strait Islander communities were involved with, and in control of, every aspect of a programme from development through to evaluation.

Inadequate funding for indigenous tobacco control and the resulting lack of programmes and services remains an issue. Sustained funding rather than short term, one off grants is also necessary if progress is to be made. Tobacco must compete with other health issues, such as alcohol misuse, that are often seen as more immediate and important, given limited funding. Increasing the prioritisation of tobacco control within Aboriginal and Torres Strait Islander health through advocacy and policy change is critical.

Increased research into the design and implementation of interventions and better evaluation of programmes is needed to help determine best practice for tobacco control in Aboriginal and Torres Strait Islander communities. The current lack of evidence for best practice means that even if communities are ready to act, they have little guidance to help increase the chance of implementing successful programmes.

\section{CONCLUSION}

Aboriginal and Torres Strait Islander people suffer disproportionately from tobacco related health conditions. The high prevalence of tobacco use and normalisation of tobacco use within this population is at least partially attributable to the process of colonisation and the subsequent low socioeconomic status of Aboriginal and Torres Strait Islander Australians.

If there are to be improvements in health outcomes related to tobacco use within Aboriginal and Torres Strait Islander communities, and subsequent reductions in the disparity between the health status of this population and other Australians, it is critical that adequately funded, well coordinated, community controlled tobacco control programmes are supported.

Tobacco Control 2003;12(Suppl II):ii5-ii8

Authors' affiliations

VL Briggs, KJ Lindorff, The Cancer Council

Victoria, Australia

RG Ivers, Menzies School of Health Research, Australia

For correspondence: KJ Lindorff, The Cancer Council Victoria, Australia;

kylie.lindorff@cancervic.org.au

\section{REFERENCES}

1 Cunningham J, Condon JR. Premature mortality in Aboriginal adults in the Northern Territory, 1979-1991. Med J Aust 1996; 165:309-12.

2 Statistics New Zealand and Ministry of Health. A picture of health. Wellington: Statistics New Zealand and Ministry of Health, 1993.

3 Stephens T, Fowler GD. Canada's Health Promotion Survey 1990: technical support. Ottawa: Minister of Supply and Services, 1993.

4 Australian Bureau of Statistics. National Aboriginal and Torres Strait Islander Survey 1994: health of indigenous Australians. Canberra: Australian Bureau of Statistics, 1996

5 Hoy WE, Norman R, Hayhurst BG, et al. A health profile of adults in a Northern Territory aboriginal community, with an emphasis on preventable morbidities [see comments]. Aust NZJ Public Health 1997;21 (2): 121-6.

6 Watson C, Fleming J, Alexander K. A survey of drug use patterns in Northern Territory Aboriginal communities 1986-1987. Darwin: Northern Territory Department of Health and Community Services, 1988.

7 Australian Institute of Health and Welfare. 1998 National Drug Strategy Household Survey: first results. Canberra: Australian Institute of Health and Welfare (Drug Statistics Series), 1999.

8 Hill DJ, White VM, Scollo MM. Smoking behaviours of Australian adults in 1995. trends and concerns. Med J Aust 1998;168:209-13.

9 Australian Institute of Health and Welfare. Australia's Health 1996. Canberra: Australian Government Publishing Service, 1996

10 McLennan W, Madden R. The Health and Welfare of Australia's Aboriginal and Torres Strait Islander Peoples. Canberra: Australian Bureau of Statistics, 1997.

11 Taylor R. Risk of premature death from smoking in 15-year-old Australians. Aust Public Health 1993;17(4):358-64.

12 Arnold-Reed DE, Holman CD, Codde J, et al. Effects of smoking and unsafe alcohol consumption on aboriginal life expectancy [letter]. Med J Aust 1998;168(2):95.

13 Bhatia K, Anderson P. An overview of Aboriginal and Torres Strait Islander health: present status and future trends. An information paper. Canberra: Australian Institute of Health and Welfare, 1995.

14 English D, Holman C, Milne E, et al. The quantification of drug caused morbidity and mortality in Australia 1995. Canberra: Australian Government Publishing Service, 1995

15 Measey M, d'Espaignet E, Cunningham J. Mortality and morbidity attributable to smoking, Northern Territory 1986-1995. Darwin: Territory Health Services, 1998.

16 Low T. Pituri - tracing the trade routes of an indigenous intoxicant. Australian Natural History 1987;22(6):257-60.

17 Macknight C. The Voyage to Marege. Melbourne: Melbourne University Press, 1976.

18 Thomson D. Notes on the smoking-pipes of North Queensland and the Northern Territory of Australia. Man 1939;39:81-91. 
19 Brady M. Roots of tobacco use among Indigenous people. Aust NZ J Public Health 2002;26(2):120-4

20 Rowse T. White flour, white power: from rations to citizenship in central Australia. Melbourne: Cambridge University Press, 1998.

21 Cunningham J. Cigarette smoking among indigenous Australians, 1994. Canberra: Australian Bureau of Statistics, 1994.

22 Australian Bureau of Statistics. 1996 Census of population and housing - Aboriginal and Torres Strait Islander people. Canberra: Australian Bureau of Statistics, 1998.

23 Australian Institute of Health and Welfare. Australia's health 2000: the seventh biennial health report of the Australian Institute of Health and Welfare. Canberra: Australian Institute of Health and Welfare, 2000.

24 Roche A, Ober C. Rethinking smoking among Aboriginal Australians: the harm

minimisation-abstinence conundrum. Health Promotion Journal of Australia 1997;7(2):128-33.
25 Lindorff K. Tobacco - time for action. Canberra: National Aboriginal Community Controlled Health Organisation, 2002

26 Commonwealth Department of Human Services and Health. National Drug Strategy Household Survey. Canberra: Australian Government Publishing Service, 1996.

27 Ivers R. Indigenous Australians and tobacco: a literature review. Darwin: Menzies School of Health Research and the Cooperative Research Centre for Aboriginal and Tropical Health, 2001

28 National Tobacco Campaign. Australia's National Tobacco Campaign: evaluation report volume one. Canberra: Commonwealth Department of Health and Aged Care, 1999.

29 Deeble J, Mathers C, Smith L, et al. Expenditures on Health Services for Aboriginal and Torres Strait Islander People. Canberra: Commonwealth Department of Health and Family Services, 1998.

30 National Health and Medical Research Council. Promoting the health of indigenous Australians - a review of the infrastructure support for Aboriginal and Torres Strait Islander health advancement. Canberra: National Health and Medical Research Council, 1996

31 Harvey D, Tsey K, Cadet-James Y, et al. An evaluation of tobacco brief intervention training in three indigenous health care settings in north Queensland. Aust N Z J Public Health 2002;26(5):426-31

32 Briggs V. Smoking and health in the Koori community. Aboriginal and Islander Health Worker Journal 1996;20:17-8.

33 Di Clemente CC, Prochaska JO, Fairhurst SK, et al. The process of smoking cessation: An analysis of precontemplation, contemplation and preparation stages of change. J Consult Clin Psycho 1991;59(2);295-304.

34 Australian Medical Association and Australian Pharmaceutical Manufacturer's Association. Indigenous smoking: issues and responses. Canberra: Australian Medical Association and Australian Pharmaceutical Manufacturer's Association, 2000.
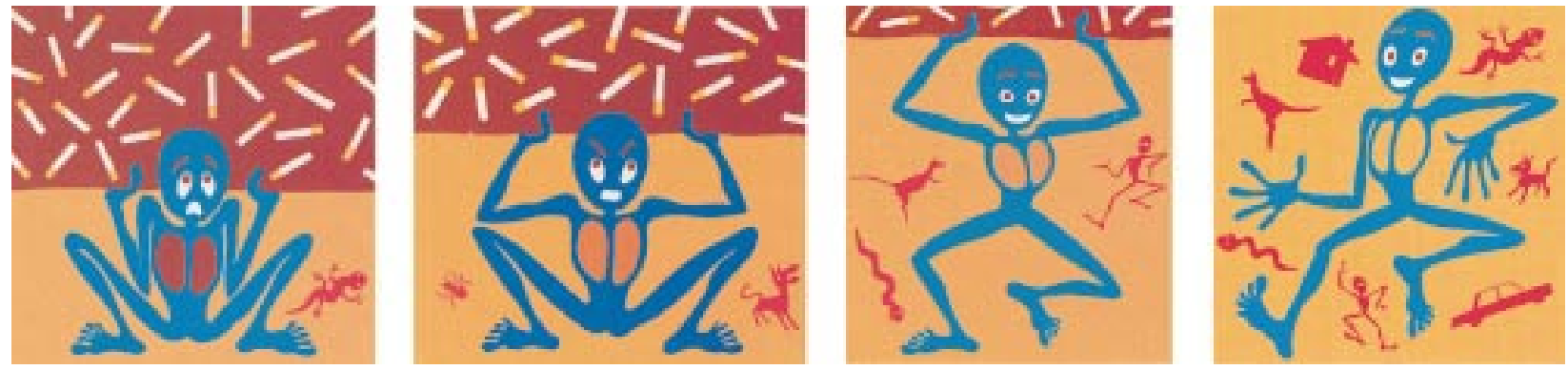

This picture shows the battle against addiction faced by indigenous Australians. Resource for Indigenous Australians. Contributed by Viki Briggs, Quit Victoria. 